\title{
Multidisciplinary approach and treatment options in right ventricular outflow tract malformations
}

This article was published in the following Dove Press journal:

Journal of Multidisciplinary Healthcare

\author{
Kristofer Skoglund' \\ Ludvig Clase ${ }^{2}$ \\ Mikael Dellborg ${ }^{3}$ \\ 'Hallands Hospital Kungsbacka, \\ Sweden, and Sahlgrenska Academy, \\ University of Gothenburg, \\ Gothenburg, Sweden; ${ }^{2}$ Sahlgrenska \\ Academy, University of Gothenburg, \\ Gothenburg, Sweden; ${ }^{3}$ Adult \\ Congenital Heart Unit, Department \\ of Medicine, Sahlgrenska University \\ Hospital, Gothenburg, Sweden
}

Background: Among patients with congenital heart disease, implantation of a valved conduit is common practice for surgical reconstruction of malformations involving the right ventricular outflow tract (RVOT). The conduit has limited durability, and treatments with surgical replacement and transcatheter pulmonary valve replacement (TPVR) are common. Previous studies indicate that TPVR, despite being a less invasive alternative, is not used for the majority of these patients.

Methods and results: This is a descriptive study of the medical records of 100 consecutive adult patients with RVOT malformations who were evaluated a total of 118 times between January 1, 2008 and December 31, 2015, at meetings of the hospital's multidisciplinary heart patient review board, in which relevant specialists make all treatment decisions on each case through a consensus process. The most common overall outcome decision was surgical conduit implantation. In 51 cases, the patient had a pre-existing conduit and, of those, 16 cases were recommended for TPVR. In seven of those 16, TPVR could not be performed, most commonly due to the risk of coronary compression or unfavorable conduit anatomy.

Conclusion: Among patients with congenital heart disease involving the RVOT, surgical conduit implantation was the main treatment both in native RVOT malformations and in the case of a pre-existing dysfunctional conduit, despite the introduction of TPVR. Although the hospital's multidisciplinary heart patient review board often recommended TPVR, it was found to be unfeasible in many cases. The main reasons were risk of coronary compression and unfavorable conduit anatomy.

Keywords: Congenital heart disease, conduit, transcatheter pulmonary valve replacement, Melody ${ }^{\circledR}$, multidisciplinary heart patient review board

\section{Introduction}

Patients with congenital heart disease constitute a large and heterogeneous group. New ways of treating this group more effectively have resulted in longer life expectancy over recent decades. ${ }^{1,2}$ Possible factors contributing to this increased survival are the development and implementation of several successful surgical procedures, improved diagnostics, centralized care, and the introduction of procedures using the percutaneous endovascular technique.

Malformations of the right ventricular outflow tract (RVOT) or pulmonary artery are common in congenital heart disease. Such malformations may be present in isolated forms or associated with concomitant lesions.

One important improvement in the care of this patient group was the introduction of surgical conduit replacement, in which a biological or human-tissue conduit
Correspondence: Kristofer Skoglund Department of Medicine, Sahlgrenska University Hospital Östra, Diagnosvägen

II, 41685 Göteborg, Sweden

$\mathrm{Tel}+46313434000$

Fax +4631191416

Email kristofer.skoglund@gu.se 
containing a valve is implanted; this enables reconstruction of the RVOT and pulmonary artery in cases where a native RVOT is missing or not amenable to repair. ${ }^{3}$ There are numerous cardiac malformations that can be treated using a conduit to reconstruct the RVOT; these include tetralogy of Fallot, transposition of the great arteries with ventricular septal defect, ${ }^{4}$ truncus arteriosus, pulmonary atresia, and double outlet right ventricle. It is also used in aortic valve stenosis implanted with a Ross procedure. ${ }^{5,6}$

Despite excellent early palliation the conduit has limited durability, and repeated operations with replacement of dysfunctional conduits are common. ${ }^{7,8}$

Transcatheter pulmonary valve replacement (TPVR), which was introduced in Sweden in 2008, is a procedure where a dysfunctional conduit is repaired. TPVR is not used in native RVOT without a conduit. A stent-mounted bovine jugular vein containing a valve (Melody valve, Medtronic, Minneapolis, MN, USA) is implanted in the conduit via the femoral vein, thus limiting the number of open heart surgeries. ${ }^{9}$ The technical implantation success rate of Melody is reported to be about $90 \%$, and it provides a high degree of significant hemodynamic improvement, resulting in reduced right ventricular pressure, reduction of pulmonary valve regurgitation, and reduction of right ventricular volume. ${ }^{10,11}$ Valve size is a limitation since the Melody valve is only available in sizes $18-22 \mathrm{~mm}$, making it unsuitable for large dilated conduits.

However, there are both short-term and long-term risks associated with TPVR. The most common short-term risks include compression of a coronary artery, conduit rupture, and vascular complications. Long-term risks include endocarditis, stent fracture, and progressive dysfunction of the TPVR such as obstruction and valve regurgitation.

To optimize decisions regarding treatment for this particular patient group, all patients considered for intervention of the RVOT or pulmonary artery are discussed at a multidisciplinary heart patient review board in which medical specialists at the hospital meet and make all treatment decisions through a consensus process. Previously published data from our group suggests that only a minority of patients theoretically eligible for TPVR actually receive the treatment. ${ }^{12}$

The aims of this study were: (1) to describe individual factors governing the decision between surgical conduit replacement and TPVR; and (2) to describe the underlying factors that prevent a planned TPVR from being performed.

\section{Materials and methods}

The study was performed at Sahlgrenska University Hospital Östra, which is one of two full-service tertiary centers in
Sweden, together accounting for all adult congenital cardiac surgery in the country. This hospital accounts for tertiary care for a population of $\sim 5$ million people, including 60-70 endovascular interventions in adults with congenital heart disease. The multidisciplinary heart patient review board consists of congenital cardiologists, adult congenital cardiothoracic surgeons, interventionists, echo specialists, and radiologists. At least one representative of each specialty is present.

We examined relevant medical records for all adult patients discussed by the multidisciplinary heart patient review board in Gothenburg between the years 2008 and 2015. Only adult patients were included (age over 18 years). Patients of relevance to our study were those with previous conduit surgery and patients with congenital or postoperative acquired malformation of the RVOT or pulmonary artery for whom further treatment was discussed. One hundred of these patients were evaluated for surgical or percutaneous treatment of the RVOT and/or pulmonary artery and were thus eligible to be included in our study. The patients were evaluated a total of 118 times. Further background and clinical information on these patients, including medical records and results from physiological and radiological examinations, was obtained from the hospital records.

Both patients with a previously implanted (pre-existing) conduit and non-conduit patients were included. Patients were assigned to one of four outcome groups depending on the decision made by the review board. These four outcome groups were: conduit surgery, TPVR, further investigation needed, and watch-and-wait. In a few cases, the review board was unable to decide between watch-and-wait and further investigation. Two of the authors (KS and $\mathrm{MD}$ ) conducted a blind reassessment of these cases before reaching a joint decision.

All calculations were created in a Microsoft Excel spreadsheet and the patients were anonymized by replacing the personal identity number with an individual numerical code for each patient. Because of the limited number of patients and a significant disparity in reported individual medical data, only descriptive statistics were used.

\section{Ethics}

The study used anonymized data for all patients and ethical approval was given by the Regional Ethics Committee of Gothenburg. Patient recruitment was based on patient participation in the national quality registry SWEDCON. SWED$\mathrm{CON}$ which is a national quality register that is approved by the proper authorities and, as most quality registers in Sweden, has been granted a waiver regarding written informed consent. 
Written information and verbal information are the only legal requirement on a national level. However, our institution has added a local requirement of written informed consent from all participants in the registry. In addition, we had a local project, examining all patients with an implanted conduit by additional magnetic resonance imaging, $\mathrm{X}$-testing and clinical outcomes. Thus, all patients were included only after approval of the projects by the proper local ethical authorities, and all patients had provided written informed consent.

\section{Results}

Between January 1, 2008 and December 31, 2015 (after TPVR was introduced at our hospital in 2008), 100 adult patients with clinical problems related to RVOT were assessed by the hospital's multidisciplinary heart patient review board, in total 118 times. The medical records were examined and results from imaging studies and functional assessments were presented. The baseline characteristics of the 100 patients at their first evaluation by the review board are given in Table 1 . Fifteen patients were discussed more than once.

The treatment decisions were divided into the predetermined four outcome groups: surgical conduit implantation, TPVR, watch-and-wait, and further investigation needed. The most common outcome decision was to offer surgical implantation of a conduit, which occurred in 63 cases. TPVR was recommended in 16 cases, watch-and-wait in 26 cases, and further investigation in 13 cases.

\section{Multidisciplinary heart patient review board decisions for patients with a pre- existing conduit}

Out of the total of 118 cases, there were 51 cases ( 47 patients, of whom 32 were male) where the patient had a pre-existing conduit. The average age was 33.1 years and 38 patients had

Table I Patient characteristics

\begin{tabular}{ll}
\hline & $\begin{array}{l}\text { All }=100, \text { Conduit } \\
(\mathbf{n})=\mathbf{4 7}\end{array}$ \\
\hline Gender & 39 women, 6I men \\
Mean age, years, (range) & $35.1(18-69)$ \\
Tetralogy of Fallot, $\mathrm{n}(\%)$ & $56(56)$ \\
Pulmonary atresia, $\mathrm{n}(\%)$ & $12(12)$ \\
Ross-operated congenital aortic valve & $8(8)$ \\
disease, $\mathrm{n}(\%)$ & \\
Transposition, $\mathrm{n}(\%)$ & $5(5)$ \\
Double outlet right ventricle, $\mathrm{n}(\%)$ & $5(5)$ \\
Pulmonary stenosis, $\mathrm{n}(\%)$ & $4(4)$ \\
Truncus arteriosus, $\mathrm{n}(\%)$ & $3(3)$ \\
Pulmonary atresia + transposition, $\mathrm{n}(\%)$ & $2(2)$ \\
Other, $\mathrm{n}(\%)$ & $5(5)$ \\
\hline
\end{tabular}

tetralogy of Fallot. They had a mean of 2.3 previous thoracic surgeries (range 1-5) and a mean of 1.4 previous conduit implantations (range 1-4).

The outcome decision in these 51 cases where the patients had an existing conduit are presented in Table 2 .

In the group with a pre-existing conduit, 15 cases were recommended for surgical conduit replacement. Within this group there was a clinically relevant conduit dysfunction in 14 cases. In one case, other surgery was planned and the decision was to add conduit replacement to the planned surgical procedure. In five of these 14 cases, the review board suggested surgical conduit replacement instead of TPVR because of an elevated risk of compressing the coronary arteries with a TPVR. In six out of 15 cases, the patient needed other cardiac surgery in addition to the conduit. Two of the 15 patients required additional cardiac surgery as well as presenting an elevated risk of coronary compression. In the remaining case, the size or other traits of the existing conduit were deemed unsuitable for TPVR.

In the group with pre-existing conduits, the board's outcome decision was watch-and-wait in 14 cases. The most common candidates for watch-and-wait, present in eight out of the 14 cases, were patients with limited or slow diseaseassociated progress of heart dysfunction and asymptomatic patients with a high level of physical functioning.

In six out of 51 cases where the patient had a pre-existing conduit, the outcome decision was that further investigations were needed to make a correct decision regarding intervention.

\section{Technical success of TPVR}

In the group with an existing conduit, a total of 16 decisions to implant TPVR were made by the multidisciplinary heart patient review board. The total number of patients was 15 , consisting of nine men and six women. The average age was 38.4 years for men and 31.2 years for women. The average number of previous surgeries was 2.7 for women and 2.5 for men.

Among the 16 decisions to attempt TPVR implantation, nine actual implantations were performed (five in women). In the last 3 years of the study only one patient was

Table 2 Decisions in pre-existing conduit group

\begin{tabular}{ll}
\hline Pre-existing conduit decisions & Cases=5 I \\
\hline Further investigations needed or watch-and-wait & 20 \\
Intervention deemed necessary & 31 \\
- Conduit surgery & 15 \\
- TPVR (transcatheter pulmonary valve replacement) & 16 \\
\hline
\end{tabular}


recommended for TPVR without actually receiving it. The remaining six failures to implant were in the first 5 years of the study. The success rate was $83 \%$ among women patients and $44 \%$ among male patients. The most common reason for not performing TPVR was that the conduit dimension was large and dilated and thus the patient's anatomy was unsuitable for TPVR ( $n=3)$. In two cases TPVR was not performed because of a high risk of coronary artery compression, as assessed during the procedure. The remaining cases were not performed because of preoperative overestimation of the severity of pulmonary dysfunction $(n=1)$, or a lack of patient compliance $(\mathrm{n}=1)$.

\section{Multidisciplinary heart patient review board decisions in patients without a pre-existing conduit}

In the group of patients without pre-existing conduits, a total of 67 cases (53 patients) were assessed. The mean age was 36.7 years, (range 19-67 years) and the mean number of previous thoracic surgeries was 1.5 . The most common malformation was tetralogy of Fallot, in 38 cases of 49 , followed by pulmonary atresia and pulmonary stenosis. In
49 cases, the patient was accepted for conduit surgery, 15 were assigned to watch-and-wait, and in three cases further evaluation was deemed necessary.

A summary of the overall findings is shown in Figure 1.

\section{Discussion}

A standardized multidisciplinary approach has been shown to be feasible in complex cardiovascular decisions. Furthermore, it enhances and contributes to stability in clinical decision-making. ${ }^{13}$ In this study, the focus was to examine the implementation of TPVR (Melody) in adult patients with malformations associated with congenital heart disease and RVOT, based on outcome decisions at the multidisciplinary heart patient review board.

We found that, even though approximately half of all the patients with RVOT or pulmonary artery malformations discussed by the review board had a pre-existing conduit, and thereby in theory should be eligible for TPVR, yet only a minority $(31 \%)$ of these patients were recommended for this treatment. The number of patients recommended for TPVR appears low in comparison to other studies. ${ }^{14}$ The main reason for the review board choosing to refrain from TPVR

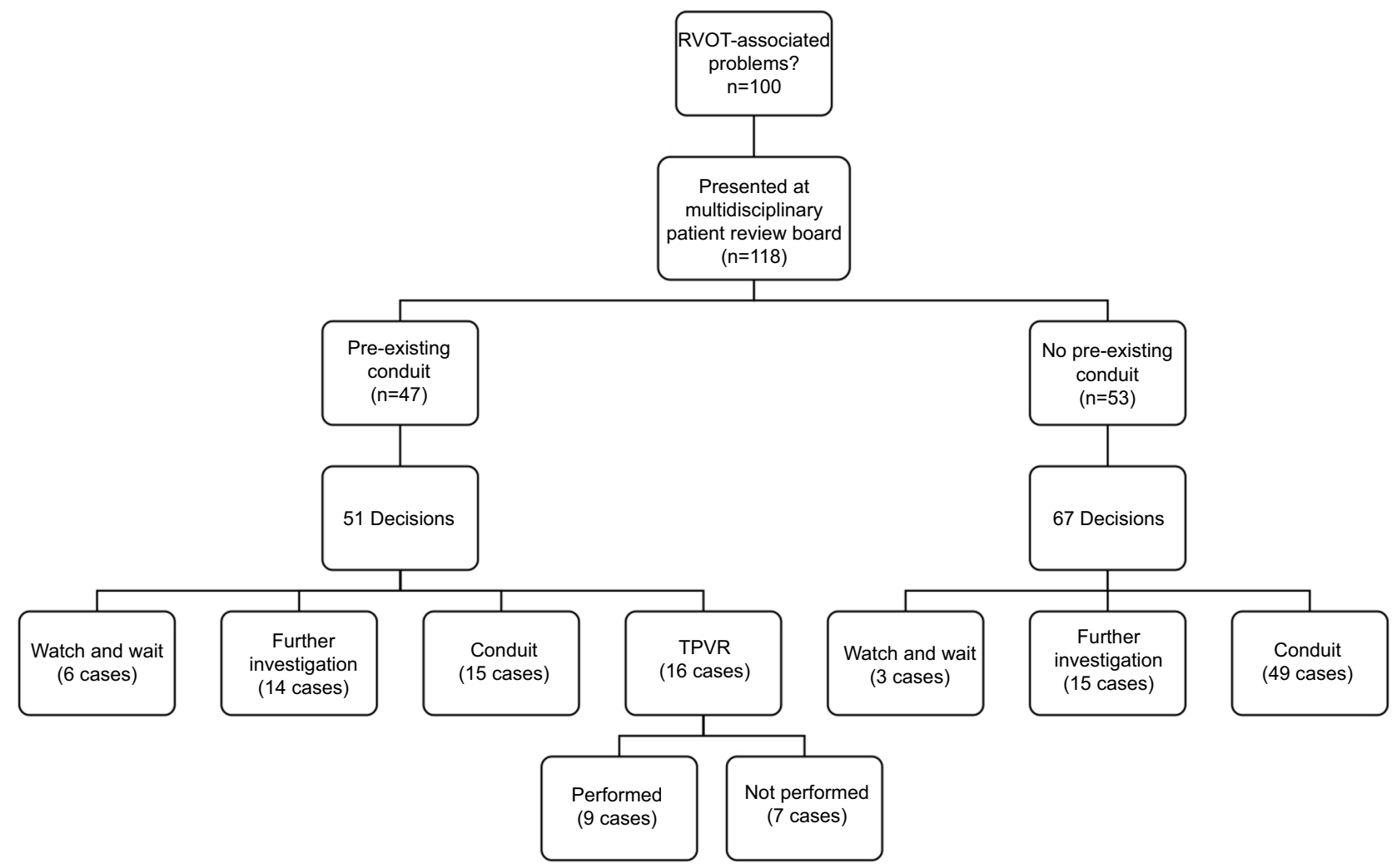

Figure I Flow chart for all 100 patients included in the study, of whom 47 had a pre-existing conduit and were thus eligible for TPVR. The final outcome was that TPVR treatment was actually performed in only nine cases.

Abbreviations: RVOT, right ventricular outflow tract; TPVR, transcatheter pulmonary valve replacement. 
was the need for concomitant cardiac surgical procedures but also concern about the risk of coronary compressions. Unsuitable anatomy of the conduit (too dilated) seemed to be less of a concern to the review board; only in one case was it given as the reason for rejecting TPVR. Thus, the risk of coronary compression, which represents the main reason for perioperative mortality in this group (incidence 1\%) appears to have been the major concern. This relatively low occurrence of decisions favoring TPVR for this group might be considered somewhat surprising, considering the fact that TPVR is a less invasive method for treating right ventricle to pulmonary artery conduit dysfunction. One explanation might be that the criteria for recommending TPVR are too narrow and conservative. The risk of coronary compressions appears to be a major concern at the review board. Is it overestimated? Is there, despite this concern, reason to proceed to invasive evaluation of coronaries in more cases, in order to enable more patients to have TPVR?

Furthermore, the selection of patients to be considered by the review board may be biased. The low rate of outcome decisions for surgery or TPVR compared to watch-and-wait or further investigation may appear too conservative; on the other hand, the multidisciplinary review board is an important forum for the discussion of complex cases when aiming for high quality of care. Limiting the number of patients to be assessed may have a negative impact on patient care.

Another interesting finding is that almost half (seven out of 16) of the cases where the outcome decision was for TPVR did not receive this treatment. This was mainly because the conduit was anatomically unsuitable for implantation but also because of a risk of coronary artery compression after invasive evaluation. The conduit was too large in three of the cases. There are reports on stenting (Russian doll technique) with multiple pre-stenting to reduce the conduit lumen size. Clearly the valve should be developed to fit large and dilated conduits in order to improve applicability. Moreover, TPVR has been conducted elsewhere using the Edwards valve (Edwards Life Sciences), which is available in larger sizes (23-26 mm and $29 \mathrm{~mm}$ for the Edwards XT) whereas the Melody ${ }^{\circledR}$ valve used in our hospital is only available in sizes $18-22 \mathrm{~mm} .{ }^{15}$ Use of this device could improve applicability. Another important aspect in this patient group is the preoperative radiological assessment at the multidisciplinary review board. This assessment did not raise concerns for the applicability of TPVR. It is possible that clinicians in some cases proceeded to attempt TPVR instead of surgery in borderline cases since the technique is less invasive and provides an attractive alternative to surgery for the patient. Another possible explanation is that the pre-procedural radiological examinations were not specifically targeted to examine conduit anatomy in detail. Moreover, in the last 3 years of the study period, only one patient with the outcome decision TPVR did not actually receive the treatment, suggesting a learning curve in selecting suitable candidates for TPVR.

Of the 51 cases with conduit dysfunction, two-thirds were considered to be in need of some kind of intervention for their problematic RVOT. Of these 31 patients, 16 were considered eligible for TPVR but only nine patients ( $18 \%$ of the total number evaluated) actually received the treatment. Even accounting for the eight patients in need of concomitant cardiac surgery, the chance of a patient with conduit failure having a TPVR was very low: less than one in four. We conclude that surgical conduit implantation and replacement of failing conduits appear to be the most applicable line of treatment despite the introduction of TPVR. New and improved TPVR suitable for patients with different anatomical variants may increase the applicability of the technique.

\section{Limitations}

The low number of available patients limited the scope for statistical analysis. Because patients had to be evaluated at the multidisciplinary heart patient review board to be included in the study, we were unable to include occasional patients managed in the clinic. The study was a single-institution study and the results obtained reflects this institution.

\section{Conclusion}

We conclude that, among patients with congenital heart disease involving the RVOT, surgical conduit implantation was the main treatment option in native RVOT as well as in pre-existing dysfunctional conduits, which theoretically makes TPVR an applicable alternative. The risk of coronary compression and unfavorable conduit anatomy were the main concerns preventing the use of TPVR in this study. Despite case discussion at a multidisciplinary heart patient review board often recommending TPVR treatment, it was not found to be applicable in many of these cases. The applicability of TPVR may increase with evolution of the technique to suit more anatomical variants.

\section{Acknowledgments}

This study was funded by ALF-LUA grants from Sahlgrenska University Hospital, grants from the Health and Technology Assessment Center at Sahlgrenska University Hospital and by a grant from the Swedish Heart and Lung Foundation. We want to thank the dedicated staff at the adult congenital heart unit in Gothenburg. 


\section{Disclosure}

The authors report no conflicts of interest in this work.

\section{References}

1. Mandalenakis Z, Rosengren A, Skoglund K, Lappas G, Eriksson P, Dellborg M. Survivorship in children and young adults with congenital heart disease in Sweden. JAMA Intern Med. 2017;177(2):224-230.

2. Moons P, Bovijn L, Budts W, Belmans A, Gewillig M. Temporal trends in survival to adulthood among patients born with congenital heart disease from 1970 to 1992 in Belgium. Circulation. 2010;122(22):2264-2272.

3. Ross DN, Somerville J. Correction of pulmonary atresia with a homograft aortic valve. Lancet. 1966;2(7479):1446-1447.

4. Rastelli GC, McGoon DC, Wallace RB. Anatomic correction of transposition of the great arteries with ventricular septal defect and subpulmonary stenosis. J Thorac Cardiovasc Surg. 1969;58(4):545-552.

5. Caldarone CA, McCrindle BW, Van Arsdell GS, et al. Independent factors associated with longevity of prosthetic pulmonary valves and valved conduits. J Thorac Cardiovasc Surg. 2000;120(6):1022-1030; discussion 1031.

6. Dearani JA, Danielson GK, Puga FJ, et al. Late follow-up of 1095 patients undergoing operation for complex congenital heart disease utilizing pulmonary ventricle to pulmonary artery conduits. Ann Thorac Surg. 2003;75(2):399-410; discussion 410-411.

7. Buber J, Assenza GE, Huang A, et al. Durability of large diameter right ventricular outflow tract conduits in adults with congenital heart disease. Int J Cardiol. 2014;175(3):455-463.
8. Skoglund K, Svensson G, Thilen U, Dellborg M, Eriksson P. Long-term outcome after right ventricle to pulmonary artery conduit surgery and reintervention. Scand Cardiovasc J. 2017;51(5):284-291.

9. Bonhoeffer P, Boudjemline Y, Saliba Z, et al. Percutaneous replacement of pulmonary valve in a right-ventricle to pulmonary-artery prosthetic conduit with valve dysfunction. Lancet. 2000;356(9239): $1403-1405$.

10. Cheatham JP, Hellenbrand WE, Zahn EM, et al. Clinical and hemodynamic outcomes up to 7 years after transcatheter pulmonary valve replacement in the US melody valve investigational device exemption trial. Circulation. 2015;131(22):1960-1970.

11. Eicken A, Ewert P, Hager A, et al. Percutaneous pulmonary valve implantation: two-centre experience with more than 100 patients. Eur Heart J. 2011;32(10):1260-1265.

12. Skoglund K, Svensson G, Thilen U, Dellborg M, Eriksson P. RV to PA conduits: impact of transcatheter pulmonary valve replacement in adults - a national register study. Scand Cardiovasc J. 2017;51(3): $153-158$.

13. Mirzada N, Ladenvall P, Hansson PO, Eriksson P, Dellborg M. Multidisciplinary management of patent foramen ovale (PFO) and cryptogenic stroke/TIA. J Multidiscip Healthc. 2013;6:357-363.

14. Steinberg ZL, Jones TK, Verrier E, Stout KK, Krieger EV, Karamlou T. Early outcomes in patients undergoing transcatheter versus surgical pulmonary valve replacement. Heart. 2017;103(18):1455-1460.

15. Kenny D, Hijazi ZM, Kar S, et al. Percutaneous implantation of the Edwards SAPIEN transcatheter heart valve for conduit failure in the pulmonary position: early phase 1 results from an international multicenter clinical trial. J Am Coll Cardiol. 2011;58(21):2248-2256.
Journal of Multidisciplinary Healthcare

\section{Publish your work in this journal}

The Journal of Multidisciplinary Healthcare is an international, peerreviewed open-access journal that aims to represent and publish research in healthcare areas delivered by practitioners of different disciplines. This includes studies and reviews conducted by multidisciplinary teams as well as research which evaluates the results or conduct of such teams or health

\section{Dovepress}

care processes in general. The journal covers a very wide range of areas and welcomes submissions from practitioners at all levels, from all over the world. The manuscript management system is completely online and includes a very quick and fair peer-review system. Visit http://www.dovepress.com/ testimonials.php to read real quotes from published authors. 